\title{
The dynamic properties of solutions for a nonlinear shallow water equation
}

Yeqin Su', Shaoyong Lai ${ }^{2}$ and Sen Ming ${ }^{3 *}$

"Correspondence: senming1987@163.com

${ }^{3}$ Department of Mathematics, North University of China, Taiyuan, China Full list of author information is available at the end of the article

\begin{abstract}
The local well-posedness for the Cauchy problem of a nonlinear shallow water equation is established. The wave-breaking mechanisms, global existence, and infinite propagation speed of solutions to the equation are derived under certain assumptions. In addition, the effects of coefficients $\lambda, \beta, a, b$, and index $k$ in the equation are illustrated.
\end{abstract}

Keywords: Local well-posedness; Wave-breaking; Global solution; Infinite propagation speed

\section{Introduction}

We aim to consider the problem

$$
\left\{\begin{array}{l}
v_{t}-v_{x x t}+\beta\left(v_{x}-v_{x x x}\right)+\lambda\left(v-v_{x x}\right)+(a+b) v^{k} v_{x} \\
\quad=b v^{k-1} v_{x} v_{x x}+a v^{k} v_{x x x}, \\
v(0, x)=v_{0}(x) .
\end{array}\right.
$$

Here $(t, x) \in \mathbb{R}^{+} \times \mathbb{R}, v(t, x)$ is fluid velocity of water waves, $\lambda \in \mathbb{R}^{+}, \beta \in \mathbb{R},(a, b) \in \mathbb{R}^{2}$, $k$ is a positive integer, $\beta\left(v-v_{x x}\right)$ is the diffusion term, $\lambda\left(v-v_{x x}\right)$ is the dissipative term, $v_{0} \in B_{p, r}^{s}(\mathbb{R})\left(s>\max \left(1+\frac{1}{p}, \frac{3}{2}\right)\right)$.

Recently, the Camassa-Holm $(\mathrm{CH})$ equation

$$
v_{t}-v_{x x t}+\beta v_{x}+3 v v_{x}=2 v_{x} v_{x x}+v v_{x x x}
$$

has attracted much attention. Equation (1.2) admits blow-up phenomena. Replacing $v$ with $v+\beta$ in Eq. (1.2), we obtain

$$
v_{t}-v_{x x t}+\beta\left(v_{x}-v_{x x x}\right)+3 v v_{x}=2 v_{x} v_{x x}+v v_{x x x} .
$$

Taking $k=1, \lambda=0, a=1, b=2$ in (1.1) gives rise to the Cauchy problem of Eq. (1.3). The solution $v$ to Eq. (1.2) is viewed as a perturbation near $\beta$ (see [20]). The properties of solutions to the problem with dispersion and dissipative terms are discovered in [15]. Mi et al. [12] investigate the dynamical properties for a generalized $\mathrm{CH}$ equation. For a related

(c) The Author(s) 2019. This article is distributed under the terms of the Creative Commons Attribution 4.0 International License (http://creativecommons.org/licenses/by/4.0/), which permits unrestricted use, distribution, and reproduction in any medium, provided you give appropriate credit to the original author(s) and the source, provide a link to the Creative Commons license, and indicate if changes were made. 
study of the $\mathrm{CH}$ equation and other related partial differential equations, one may refer to references $[3,7,11,14,16]$.

Taking $k=1, \lambda=\beta=0, a=1, b=3$ in (1.1) yields the Degasperis-Procesi equation

$$
v_{t}-v_{x x t}+4 v v_{x}=3 v_{x} v_{x x}+v v_{x x x} .
$$

The formation of singularity for solutions to (1.4) is discovered in [17]. Lai and Wu [10] study the local well-posedness for the Cauchy problem of

$$
v_{t}-v_{x x t}+\beta v_{x}+(a+b) v_{x}=b v_{x} v_{x x}+a v v_{x x x}
$$

where $\beta, a, b \in \mathbb{R}$.

Taking $k=2, \lambda=\beta=0, a=1, b=3$ in (1.1), we obtain the Novikov equation

$$
v_{t}-v_{x x t}+4 v^{2} v_{x}=3 v v_{x} v_{x x}+v^{2} v_{x x x} .
$$

Guo [4] studies the persistence properties of solutions to the $\mathrm{CH}$-type equation. Fu and $\mathrm{Qu}$ [2] discover blow-up of solutions to Eq. (1.6) in $H^{s}(\mathbb{R})\left(s>\frac{5}{2}\right)$. The peakon solutions to the Novikov equation are established in [6].

Himonas and Thompson [8] discover persistence properties for solutions if $\lambda=\beta=$ $0, a=1$ in (1.1). The behaviors of solutions [5], global existence of solutions for $a=1$ [9], and infinite propagation speed of solutions $[9,19]$ to the problems are investigated. We extend parts of results in $[9,10,13,18,19]$.

Let $s \in \mathbb{R}, T>0, p \in[1, \infty]$ and $r \in[1, \infty]$. Thus we set

$$
E_{p, r}^{s}(T)= \begin{cases}C\left([0, T] ; B_{p, r}^{s}(\mathbb{R})\right) \cap C^{1}\left([0, T] ; B_{p, r}^{s-1}(\mathbb{R})\right), & 1 \leq r<\infty \\ L^{\infty}\left([0, T] ; B_{p, \infty}^{s}(\mathbb{R})\right) \cap \operatorname{Lip}\left([0, T] ; B_{p, \infty}^{s-1}(\mathbb{R})\right), & r=\infty\end{cases}
$$

Letting $P_{1}(D)=-\partial_{x}\left(1-\partial_{x}^{2}\right)^{-1}, P_{2}(D)=\left(1-\partial_{x}^{2}\right)^{-1}$, problem $(1.1)$ is turned into

$$
\left\{\begin{aligned}
v_{t}+\left(a v^{k}+\beta\right) v_{x}= & P_{1}(D)\left[\frac{b}{k+1} v^{k+1}+\frac{3 a k-b}{2} v^{k-1} v_{x}^{2}\right] \\
& +P_{2}(D)\left[\frac{(k-1)(a k-b)}{2} v^{k-2} v_{x}^{3}-\lambda v\right], \\
v(0, x)=v_{0}(x) . &
\end{aligned}\right.
$$

Now we summarize the main results in this paper.

Theorem 1.1 Suppose $1 \leq r, p \leq \infty, v_{0} \in B_{p, r}^{s}(\mathbb{R})\left(s>\max \left(1+\frac{1}{p}, \frac{3}{2}\right)\right)$. Then solution $v \in$ $E_{p, r}^{s}(T)$ to problem (1.1) is locally well-posed for certain $T>0$.

Theorem 1.2 Suppose $1 \leq r, p \leq \infty, v_{0} \in B_{p, r}^{s}(\mathbb{R})\left(s>\max \left(1+\frac{1}{p}, \frac{3}{2}\right)\right), t \in[0, T]$. Then $a$ solution $v$ to problem (1.1) blows up in finite time if and only if

$$
\int_{0}^{t}\left(1+\left\|v_{x}\right\|_{L^{\infty}}\right)^{k} d \tau=\infty
$$


Theorem 1.3 Suppose $b=a(k+1)$ and $v_{0} \in H^{s}(\mathbb{R})\left(s>\frac{3}{2}\right), t \in[0, T]$. Then a solution $v$ to problem (1.1) blows up in finite time if and only if

$$
\lim _{t \rightarrow T^{-}} \inf _{x \in \mathbb{R}} v_{x}(t, x)=-\infty
$$

Theorem 1.4 Suppose $b=a(k+1)$ and $v_{0} \in H^{s}(\mathbb{R})(s \geq 2)$ satisfies $\left\|v_{0}-v_{0, x x}\right\|_{L^{2}}<$ $\frac{4 \lambda}{|a|(k+2)\left\|\nu_{0}\right\|_{H^{1}}^{k-1}}$. Then there exists a global solution to problem $(1.1)$ in $H^{s}(\mathbb{R})(s \geq 2)$.

Theorem 1.5 Assume $v_{0} \in H^{s}(\mathbb{R})(s \geq 2), n_{0}(x)=v_{0}-v_{0, x x} \neq 0$ for all $x \in \mathbb{R},\left\|n_{0}\right\|_{L^{2}}<$ $\left(\frac{2^{k+1} \lambda}{|a k-2 b|}\right)^{\frac{1}{k}}$ and $b \neq \frac{a k}{2}$. Then a solution $v$ to problem $(1.1)$ is global in $H^{s}(\mathbb{R})(s \geq 2)$.

Theorem 1.6 Assume $a>0$ and let $v_{0} \in H^{s}(\mathbb{R})\left(s>\frac{5}{2}\right)$ be compactly supported in $\left[a_{0}, b_{0}\right]$, $t \in[0, T]$. Suppose $k$ is a positive odd number and $b=a k$, or $k=1,0<b<3 a$. Then, the solution $v(t, x)$ to (1.1) satisfies

$$
v(t, x)=\frac{1}{2} L_{+}(t) e^{-x} \quad \text { for } x \geq p\left(t, b_{0}\right), \quad v(t, x)=\frac{1}{2} L_{-}(t) e^{x} \quad \text { for } x \leq p\left(t, a_{0}\right),
$$

where $L_{+}(t)$ and $L_{-}(t)$ are continuous non-vanishing functions given in (4.1). What is more, $L_{+}(t)>0, L_{-}(t)<0$ for $t \in[0, T]$. In particular, if $k=1, b=2 a$ or $b=\frac{a}{2}$, then $L_{+}(t) \leq C_{3} e^{(\beta-\lambda) t}$ and $\left|L_{-}(t)\right| \leq C_{4} e^{-(\beta+\lambda) t}$.

Remark 1.1 Problem (1.1) is local well-posed in $B_{p, r}^{s}(\mathbb{R})\left(s>\max \left(\frac{3}{2}, 1+\frac{1}{p}\right)\right) \cdot\|v(t)\|_{H^{1}(\mathbb{R})}$ is bounded if $b=a(k+1)$. Also $\|v(t)\|_{H^{2}(\mathbb{R})}$ is bounded if $b=\frac{a k}{2}$. Theorem 1.2 improves the result of Theorem 5.1 in [19]. Theorem 1.3 implies that wave-breaking for a solution $v$ occurs if its slope is unbounded. This result improves Theorem 3.1 in [18] and Theorem 5.6 in [19]. From Theorems 1.4, 1.5, and 1.6, we deduce that $\lambda, \beta, a, b$, and $k$ are related to global existence and infinite propagation speed of the solutions. Parts of results in $[9,10$, $13,18,19]$ are extended.

\section{Proof of Theorem 1.1}

We prove Theorem 1.1 in following five steps.

Step 1 . Let $v^{0}=0$. Let $\left(v^{i}\right)_{i \in \mathbb{N}} \in C\left(\mathbb{R}^{+} ; B_{p, r}^{\infty}\right)$ be smooth and satisfy

$$
\left\{\begin{array}{l}
\left(\partial_{t}+\left(a\left(v^{i}\right)^{k}+\beta\right) \partial_{x}\right) v^{i+1}=G, \\
v^{i+1}(0, x)=v_{0}^{i+1}=S_{i+1} v_{0},
\end{array}\right.
$$

and suppose

$$
\begin{aligned}
G= & P_{1}(D)\left[\frac{b}{k+1}\left(v^{i}\right)^{k+1}+\frac{3 a k-b}{2}\left(v^{i}\right)^{k-1}\left(v^{i}\right)_{x}^{2}\right] \\
& +P_{2}(D)\left[\frac{(k-1)(a k-b)}{2}\left(v^{i}\right)^{k-2}\left(v^{i}\right)_{x}^{3}-\lambda v^{i}\right] .
\end{aligned}
$$

We see $S_{i+1} v_{0} \in B_{p, r}^{\infty}$. Then the solution $v^{i} \in C\left(\mathbb{R}^{+} ; B_{p, r}^{\infty}\right)$ in $(2.1)$ is global for all $i \in \mathbb{N}$ by Lemma 2.5 in [13]. 
Step 2. It is derived from Lemma 2.4 in [13] that

$$
\begin{aligned}
\left\|v^{i+1}\right\|_{B_{p, r}^{s}} \leq & e^{C_{1} \int_{0}^{t}\left\|\left(v^{i}(\tau)\right)^{k}\right\|_{B_{p, r}^{s}} d \tau} \\
& \times\left[\left\|v_{0}\right\|_{B_{p, r}^{s}}+\int_{0}^{t} e^{-C_{1} \int_{0}^{\tau}\left\|\left(v^{i}(\xi)\right)^{k}\right\|_{B_{p, r}^{s}} d \xi}\|G(\tau, \cdot)\|_{B_{p, r}^{s}} d \tau\right] .
\end{aligned}
$$

The notation $a \lesssim b$ means $a \leq C b$ for a certain positive constant $C$. We acquire the estimates

$$
\|G(t, x)\|_{B_{p, r}^{s}} \lesssim\left(\left\|v^{i}\right\|_{B_{p, r}^{s}}+1\right)^{k}\left\|v^{i}\right\|_{B_{p, r}^{s}}
$$

That is,

$$
\begin{aligned}
& \left\|v^{i+1}\right\|_{B_{p, r}^{s}} \leq C_{2} \cdot e^{C_{2} \int_{0}^{t}\left(\left\|v^{i}(\tau)\right\|_{B_{p, r}^{s}}+1\right)^{k} d \tau}\left[\left\|v_{0}\right\|_{B_{p, r}^{s}}\right. \\
& \left.+\int_{0}^{t} e^{-C_{2} \int_{0}^{\tau}\left(\left\|v^{i}(\xi)\right\|_{B_{p, r}^{s}}+1\right)^{k} d \xi}\left(\left\|v^{i}\right\|_{B_{p, r}^{s}}+1\right)^{k}\left\|v^{i}\right\|_{B_{p, r}^{s}} d \tau\right] \text {. }
\end{aligned}
$$

One may find certain $T>0$ which satisfies $2 k C_{2}^{k+1}\left(1+\left\|v_{0}\right\|_{B_{p, r}^{s}}\right)^{k} T<1$ and

$$
\left(1+\left\|v^{i}(t)\right\|_{B_{p, r}^{s}}\right)^{k} \leq \frac{C_{2}^{k}\left(1+\left\|v_{0}\right\|_{B_{p, r}^{s}}\right)^{k}}{1-2 k C_{2}^{k+1}\left(1+\left\|v_{0}\right\|_{B_{p, r}^{s}}\right)^{k} t} .
$$

Further, we deduce

$$
\left(1+\left\|v^{i+1}(t)\right\|_{B_{p, r}^{s}}\right)^{k} \leq \frac{C_{2}^{k}\left(1+\left\|v_{0}\right\|_{B_{p, r}^{s}}\right)^{k}}{1-2 k C_{2}^{k+1}\left(1+\left\|v_{0}\right\|_{B_{p, r}^{s}}\right)^{k} t}
$$

which implies that $\left(v^{i}\right)_{i \in \mathbb{N}}$ is uniformly bounded in $E_{p, r}^{s}(T)$.

Step 3. Let $m, n \in \mathbb{N}$. From (2.1), we deduce that

$$
\begin{aligned}
\left(\partial_{t}+\right. & \left.\left(a\left(v^{m+n}\right)^{k}+\beta\right) \partial_{x}\right)\left(v^{m+n+1}-v^{m+1}\right) \\
= & -a\left(\left(v^{m+n}\right)^{k}-\left(v^{m}\right)^{k}\right) \partial_{x} v^{m+1} \\
& +P_{1}(D)\left[\frac{b}{k+1}\left(\left(v^{m+n}\right)^{k+1}-\left(v^{m}\right)^{k+1}\right)\right] \\
& +P_{1}(D)\left[\frac{3 a k-b}{2}\left(\left(v^{m+n}\right)^{k-1}\left(v^{m+n}\right)_{x}^{2}-\left(v^{m}\right)^{k-1}\left(v^{m}\right)_{x}^{2}\right)\right] \\
& +P_{2}(D)\left[\frac{(k-1)(a k-b)}{2}\left(\left(v^{m+n}\right)^{k-2}\left(v^{m+n}\right)_{x}^{3}-\left(v^{m}\right)^{k-2}\left(v^{m}\right)_{x}^{3}\right)\right] \\
& +P_{2}(D)\left[-\lambda\left(v^{m+n}-v^{m}\right)\right] .
\end{aligned}
$$

Using Lemma 2.4 in [13] yields

$$
\left\|v^{m+n+1}-v^{m+1}\right\|_{B_{p, r}^{s-1}}
$$




$$
\begin{aligned}
\leq & e^{C \int_{0}^{t}\left\|v^{m+n}\right\|_{B_{p, r}^{s}}^{k} d \tau}\left[\left\|v_{0}^{m+n+1}-v_{0}^{m+1}\right\|_{B_{p, r}^{s-1}}+C \times \int_{0}^{t} e^{-C \int_{0}^{\tau}\left\|v^{m+n}\right\|_{B_{p, r}^{s}}^{k} d \xi}\right. \\
& \left.\times\left(\left\|v^{m+n}-v^{m}\right\|_{B_{p, r}^{s s}}\left(\left\|v^{m}\right\|_{B_{p, r}^{s}}+\left\|v^{m+n}\right\|_{B_{p, r}^{s}}+\left\|v^{m+1}\right\|_{B_{p, r}^{s}}+1\right)^{k}\right) d \tau\right] .
\end{aligned}
$$

We note that the initial values satisfy

$$
v_{0}^{m+n+1}-v_{0}^{m+1}=\sum_{q=m+1}^{m+n} \Delta_{q} v_{0}
$$

One may find a constant $C_{T_{1}}$ independent of $m$ to satisfy

$$
\left\|v^{m+n+1}-v^{m+1}\right\|_{L^{\infty}\left([0, T] ; B_{p, r}^{s-1}\right)} \leq C_{T_{1}} 2^{-m}
$$

We obtain the desired results.

Step 4. Following the discussions in Step 4 in Sect. 3.1 in [13], one derives that $v \in E_{p, r}^{s}(T)$, which is continuous.

Step 5. (Proof of the uniqueness). Suppose $1 \leq r, p \leq \infty, s>\max \left(\frac{3}{2}, 1+\frac{1}{p}\right)$. Assume $v^{1}$ and $v^{2}$ satisfy (1.7) with $v_{0}^{1}, v_{0}^{2} \in B_{p, r}^{s}, v^{1}, v^{2} \in L^{\infty}\left([0, T] ; B_{p, r}^{s}\right) \cap C\left([0, T] ; B_{p, r}^{s-1}\right)$. We write $v^{12}=$ $v^{1}-v^{2}$. Then

$$
v^{12} \in L^{\infty}\left([0, T] ; B_{p, r}^{s}\right) \cap C\left([0, T] ; B_{p, r}^{s-1}\right),
$$

which results in

$$
\left\{\begin{array}{l}
\partial_{t} v^{12}+\left(a\left(v^{1}\right)^{k}+\beta\right) \partial_{x} v^{12}=-a\left(\left(v^{1}\right)^{k}-\left(v^{2}\right)^{k}\right) \partial_{x} v^{2}+G_{1} \\
v^{12}(0, x)=v_{0}^{12}=v_{0}^{1}-v_{0}^{2}
\end{array}\right.
$$

where

$$
\begin{aligned}
G_{1}= & P_{1}(D)\left[\frac{b}{k+1}\left(\left(v^{1}\right)^{k+1}-\left(v^{2}\right)^{k+1}\right)\right] \\
& +P_{1}(D)\left[\frac{3 a k-b}{2}\left(\left(v^{1}\right)^{k-1}\left(v^{1}\right)_{x}^{2}-\left(v^{2}\right)^{k-1}\left(v^{2}\right)_{x}^{2}\right)\right] \\
& +P_{2}(D)\left[\frac{(k-1)(a k-b)}{2}\left(\left(v^{1}\right)^{k-2}\left(v^{1}\right)_{x}^{3}-\left(v^{2}\right)^{k-2}\left(v^{2}\right)_{x}^{3}\right)-\lambda v^{12}\right] .
\end{aligned}
$$

Using Lemma 2.4 in [13], we derive the estimates

$$
\begin{aligned}
& e^{-C \int_{0}^{t}\left\|v^{1}\right\|_{B_{p, r}^{s}}^{k} d \tau}\left\|v^{12}\right\|_{B_{p, r}^{s-1}} \\
& \leq\left\|v_{0}^{12}\right\|_{B_{p, r}^{s-1}} \\
& \quad+C \int_{0}^{t} e^{-C \int_{0}^{\tau}\left\|v^{1}\right\|_{B_{p, r}^{s}}^{k} d \xi}\left\|v^{12}\right\|_{B_{p, r}^{s-1}}\left(\left\|v^{1}\right\|_{B_{p, r}^{s}}+\left\|v^{2}\right\|_{B_{p, r}^{s}}+1\right)^{k} d \tau,
\end{aligned}
$$

which finishes the proof of the uniqueness. 
Remark 2.1 Suppose $b=a(k+1), 1 \leq r, p \leq \infty, v_{0} \in B_{p, r}^{s}(\mathbb{R})\left(s>\max \left(1+\frac{1}{p}, \frac{3}{2}\right)\right), t \in[0, T]$. Then, the solution $v$ to (1.1) satisfies

$$
\|v(t)\|_{H^{1}} \leq\left\|v_{0}\right\|_{H^{1}}
$$

\section{Proofs of Theorems 1.2, 1.3, 1.4, and 1.5}

\subsection{Proof of Theorem 1.2}

Taking advantage of the operator $\Delta_{q}$ to (1.7) yields

$$
\left(\partial_{t}+\left(a v^{k}+\beta\right) \partial_{x}\right) \Delta_{q} v=a\left[v^{k}, \Delta_{q}\right] \partial_{x} v+\Delta_{q} G_{2}(t, x),
$$

where

$$
\begin{aligned}
G_{2}(t, x)= & P_{1}(D)\left[\frac{b}{k+1} v^{k+1}+\frac{3 a k-b}{2} v^{k-1} v_{x}^{2}\right] \\
& +P_{2}(D)\left[\frac{(k-1)(a k-b)}{2} v^{k-2} v_{x}^{3}-\lambda v\right] .
\end{aligned}
$$

Applying Lemma 2.3 in [13] gives rise to the estimates

$$
\left\|a\left[v^{k}, \Delta_{q}\right] \partial_{x} v\right\|_{B_{p, r}^{s}} \lesssim\left\|v_{x}\right\|_{L^{\infty}}^{k}\|v\|_{B_{p, r}^{s}}
$$

and

$$
\left\|G_{2}(t, x)\right\|_{B_{p, r}^{s}} \lesssim\left(\left\|v_{x}\right\|_{L^{\infty}}^{k}+1\right)\|v\|_{B_{p, r}^{s}} .
$$

We derive that

$$
\|v(t)\|_{B_{p, r}^{s}} \lesssim\left\|v_{0}\right\|_{B_{p, r}^{s}}+\int_{0}^{t}\left(1+\left\|v_{x}(\tau)\right\|_{L^{\infty}}\right)^{k}\|v(\tau)\|_{B_{p, r}^{s}} d \tau
$$

That is,

$$
\|v(t)\|_{B_{p, r}^{s}} \lesssim\left\|v_{0}\right\|_{B_{p, r}^{s}} e^{\int_{0}^{t}\left(1+\left\|v_{x}(\tau)\right\|_{L} \infty\right)^{k} d \tau}
$$

Letting $t \in\left[0, T^{*}\right], T^{*}<\infty$ and

$$
\int_{0}^{t}\left(1+\left\|v_{x}(\tau)\right\|_{L^{\infty}}\right)^{k} d \tau<\infty
$$

we see that $\left\|v\left(T^{*}\right)\right\|_{B_{p, r}^{s}}$ is bounded by using (3.2). It yields a contradiction, ending the proof. From Remark 2.1, we obtain a blow-up result.

Remark 3.1 If assumption $b=a(k+1)$ is added into Theorem 1.2, then condition in (1.8) is changed into

$$
\int_{0}^{t}\left(1+\left\|v_{x}\right\|_{L^{\infty}}\right)^{2} d \tau=\infty
$$




\subsection{Proof of Theorem 1.3}

We only need to prove Theorem 1.3 with $s=2$ by density argument. Take $b=a(k+1)$. It is deduced from (1.1) that

$$
\frac{1}{2} \frac{d}{d t} \int_{\mathbb{R}}\left(v^{2}+v_{x}^{2}\right) d x+\int_{\mathbb{R}} \lambda\left(v^{2}+v_{x}^{2}\right) d x=0
$$

which results in

$$
\frac{1}{2} \frac{d}{d t} \int_{\mathbb{R}}\left(v^{2}+v_{x}^{2}\right) d x \leq 0
$$

A direct calculation shows that

$$
\begin{aligned}
\frac{1}{2} & \frac{d}{d t} \int_{\mathbb{R}}\left(v_{x}^{2}+v_{x x}^{2}\right) d x \\
= & a(k+2) \int_{\mathbb{R}} v^{k} v_{x} v_{x x} d x-\int_{\mathbb{R}} \lambda\left(v_{x}^{2}+v_{x x}^{2}\right) d x \\
& -\int_{\mathbb{R}}\left[a(k+1) v^{k-1} v_{x} v_{x x}^{2}+a v^{k} v_{x x x} v_{x x}\right] d x
\end{aligned}
$$

Let $T<\infty$ and $v_{x}(t, x) \geq-M$ for a certain $M>0$. We come to the estimate

$$
\|v(t)\|_{H^{2}} \leq\left\|v_{0}\right\|_{H^{2}} e^{\left(1+M+\left\|v_{0}\right\|_{H^{1}}\right)^{k} t}, \quad \text { for all } t \in[0, T],
$$

which yields a contradiction.

\subsection{Proof of Theorem 1.4}

We take $n=v-v_{x x}$. The first equation in (1.1) is written in the form

$$
n_{t}+\beta n_{x}+\lambda n+b v^{k-1} v_{x} n+a v^{k} n_{x}=0
$$

We see $b=a(k+1)$ in Theorem 1.4. Multiplying (3.7) by $n$ and applying (3.6) gives rise to

$$
\frac{1}{2} \frac{d}{d t} \int_{\mathbb{R}} n^{2} d x+\lambda \int_{\mathbb{R}} n^{2} d x \lesssim \frac{|a|(k+2)}{4}\left\|v_{0}\right\|_{H^{1}}^{k-1}\|n\|_{L^{2}}^{3}
$$

Taking $\lambda_{1}=2 \lambda$ and $M_{1}=\frac{|a|(k+2)}{2}\left\|\nu_{0}\right\|_{H^{1}}^{k-1}$, we have

$$
\frac{d}{d t}\|n\|_{L^{2}}^{2}+\lambda_{1}\|n\|_{L^{2}}^{2} \leq M_{1}\left(\|n\|_{L^{2}}^{2}\right)^{\frac{3}{2}}
$$

It follows that $\|n\|_{L^{2}} \leq e^{-\frac{1}{2} \lambda_{1} t}\left(\frac{1}{\left\|n_{0}\right\|_{L^{2}}}-\frac{M_{1}}{\lambda_{1}}\right)^{-1}$ if $\left\|n_{0}\right\|_{L^{2}}<\frac{\lambda_{1}}{M_{1}}$. Then

$$
\left\|v_{x}\right\|_{L^{\infty}} \leq\|n\|_{L^{2}} \leq C_{2}(T)
$$

Using Theorem 1.3, we end the proof. 


\subsection{Proof of Theorem 1.5}

We investigate problem

$$
\left\{\begin{array}{l}
\frac{d}{d t} p(t, x)=a v^{k}(t, p(t, x))+\beta \\
p(0, x)=x
\end{array}\right.
$$

where $(t, x) \in(0, T) \times \mathbb{R}$.

Lemma 3.1 ([1]) Let $v \in C\left([0, T] ; H^{s}(\mathbb{R})\right) \cap C^{1}\left([0, T] ; H^{s-1}(\mathbb{R})\right)(s \geq 2),(t, x) \in[0, T] \times \mathbb{R}$. It follows that $p \in C^{1}([0, T] \times \mathbb{R}, \mathbb{R})$ to $(3.8)$ is unique and

$$
p_{x}(t, x)=e^{\int_{0}^{t} a k v^{k-1} v_{x}(\tau, p(\tau, x)) d \tau}
$$

Lemma 3.2 Let $v_{0} \in H^{s}(\mathbb{R})(s \geq 2),(t, x) \in[0, T] \times \mathbb{R}$. Then

$$
n(t, p)\left(p_{x}\right)^{\frac{b}{a k}}(t, x)=n_{0} e^{-\lambda t}
$$

Moreover, $\|n\|_{L^{\frac{a k}{b}}}=e^{-\lambda t}\left\|n_{0}\right\|_{L^{\frac{a k}{b}}}$. If $b=\frac{a k}{2}$, it holds that

$$
\|n\|_{L^{2}}=e^{-\lambda t}\left\|n_{0}\right\|_{L^{2}}
$$

Proof From (3.10), we acquire that

$$
\frac{d}{d t}\left[n(t, p)\left(p_{x}\right)^{\frac{b}{a k}}\right]=-\lambda n\left(p_{x}\right)^{\frac{b}{a k}}
$$

That is,

$$
n(t, p)\left(p_{x}\right)^{\frac{b}{a k}}=e^{-\lambda t} n_{0}(x)
$$

A direct computation gives rise to

$$
\left\|e^{-\lambda t} n_{0}(x)\right\|_{L^{\frac{a k}{b}}}=\|n\|_{L^{\frac{a k}{b}}} .
$$

We note $b=\frac{a k}{2}$. Thus we get (3.11).

Proof of Theorem 1.5 Multiplying (3.7) by $n e^{2 \lambda t}$, we come to

$$
\frac{d}{d t}\left(e^{2 \lambda t} \int_{\mathbb{R}} n^{2} d x\right)=(a k-2 b) e^{2 \lambda t} \int_{\mathbb{R}} n^{2} v^{k-1} v_{x} d x
$$

We derive that

$$
\frac{d}{d t}\left(e^{2 \lambda t} \int_{\mathbb{R}} n^{2} d x\right) \leq \frac{|a k-2 b|}{2^{k}} e^{-k \lambda t}\left[e^{2 \lambda t} \int_{\mathbb{R}} n^{2} d x\right]^{\frac{k+2}{2}}
$$


Let $h(t)=e^{2 \lambda t} \int_{\mathbb{R}} n^{2} d x$. Bearing in mind that $n_{0}(x) \neq 0, x \in \mathbb{R}$ and (3.10), one deduces that $h(t)$ is positive. Then

$$
\frac{d}{d t}[h(t)]^{-\frac{k}{2}} \geq-\frac{k}{2} \frac{|a k-2 b|}{2^{k}} e^{-k \lambda t} .
$$

Using the assumption $n_{0}(x) \neq 0, b \neq \frac{a k}{2},\left\|n_{0}\right\|_{L^{2}}<\left(\frac{2^{k+1} \lambda}{|a k-2 b|}\right)^{\frac{1}{k}}$, we have $[h(0)]^{-\frac{k}{2}}-\frac{|a k-2 b|}{2^{k+1} \lambda}>0$. We obtain the inequality

$$
\left(e^{2 \lambda t} \int_{\mathbb{R}} n^{2} d x\right)^{\frac{k}{2}} \leq\left[\left\|n_{0}\right\|_{L^{2}}^{-k}-\frac{|a k-2 b|}{2^{k+1} \lambda}\right]^{-1} .
$$

Consequently, we have the estimate

$$
\left\|v_{x}\right\|_{L^{\infty}} \leq\|n\|_{L^{2}} \leq e^{-\lambda t}\left[\left\|n_{0}\right\|_{L^{2}}^{-k}-\frac{|a k-2 b|}{2^{k+1} \lambda}\right]^{-\frac{1}{k}}
$$

Applying Theorem 1.3, we complete the proof.

We give a global existence result.

Lemma 3.3 Let $b=a(k+1)$ or $b=\frac{a k}{2}, v_{0} \in H^{s}(\mathbb{R})(s \geq 2)$. Assume $n_{0}=v_{0}-v_{0, x x}$ does not change sign. It holds that a solution $v(t, x)$ to problem (1.1) exists globally.

Proof One may assume $n_{0}(x)>0$. We use Lemma 3.2 to derive that $n>0$. Thus

$$
v(t, x)=\int_{\mathbb{R}} \frac{1}{2} e^{-|x-\xi|} n(t, \xi) d \xi \geq 0 .
$$

That is,

$$
v(t, x)=\frac{1}{2} e^{-x} \int_{-\infty}^{x} e^{\xi} n(t, \xi) d \xi+\frac{1}{2} e^{x} \int_{x}^{\infty} e^{-\xi} n(t, \xi) d \xi .
$$

We conclude that

$$
v_{x}(t, x)=-\frac{1}{2} e^{-x} \int_{-\infty}^{x} e^{\xi} n(t, \xi) d \xi+\frac{1}{2} e^{x} \int_{x}^{\infty} e^{-\xi} n(t, \xi) d \xi
$$

Hence $\left|v_{x}\right| \leq v$.

Applying $b=a(k+1)$ and recalling Remark 2.1, we derive

$$
\left|v_{x}\right| \leq|v| \lesssim\|v(t)\|_{H^{1}} \lesssim\left\|v_{0}\right\|_{H^{1}}
$$

Taking advantage of $b=\frac{a k}{2}$ and using Lemma 3.2 results in

$$
\left|v_{x}\right| \leq|v| \lesssim\|n\|_{L^{2}} \lesssim\left\|n_{0}\right\|_{L^{2}}
$$

Combining (3.18) or (3.19) with Theorem 1.2, we obtain the desired results. 


\section{Proof of Theorem 1.6}

Note that $a>0$. Using supp $v_{0}(x) \subset\left[a_{0}, b_{0}\right]$, we derive that $\operatorname{supp} v_{0}(x) \subset\left[p\left(t, a_{0}\right), p\left(t, b_{0}\right)\right]$. Applying Lemma 3.2 yields that $\operatorname{supp} n(t, x) \subset\left[p\left(t, a_{0}\right), p\left(t, b_{0}\right)\right], t \in[0, T]$.

Let

$$
L_{+}(t)=\int_{p\left(t, a_{0}\right)}^{p\left(t, b_{0}\right)} e^{\xi} n(t, \xi) d \xi, \quad L_{-}(t)=\int_{p\left(t, a_{0}\right)}^{p\left(t, b_{0}\right)} e^{-\xi} n(t, \xi) d \xi
$$

From (3.16) and (4.1), we have

$$
\begin{aligned}
v(t, x)= & \frac{1}{2} e^{-x}\left(\int_{-\infty}^{p\left(t, a_{0}\right)}+\int_{p\left(t, a_{0}\right)}^{p\left(t, b_{0}\right)}+\int_{p\left(t, b_{0}\right)}^{x}\right) e^{\xi} n(t, \xi) d \xi \\
& +\frac{1}{2} e^{x} \int_{x}^{\infty} e^{-\xi} n(t, \xi) d \xi \\
= & \frac{1}{2} e^{-x} L_{+}(t), \quad x>p\left(t, b_{0}\right) .
\end{aligned}
$$

We derive $v=\frac{1}{2} e^{x} L_{-}(t)$ if $x<p\left(t, a_{0}\right)$. Combining (3.17) with (4.2) gives rise to

$$
v=-v_{x}=v_{x x}=\frac{1}{2} e^{-x} L_{+}(t), \quad x>p\left(t, b_{0}\right)
$$

and

$$
v=v_{x}=v_{x x}=\frac{1}{2} e^{x} L_{-}(t), \quad x<p\left(t, a_{0}\right) .
$$

An application of (4.1) leads to the identity

$$
L_{+}(0)=\int_{a_{0}}^{b_{0}} e^{\xi} n_{0}(\xi) d \xi=0 .
$$

A direct calculation shows

$$
\begin{aligned}
\frac{d}{d t} L_{+}(t)= & \int_{-\infty}^{\infty} e^{\xi} n_{t}(t, \xi) d \xi \\
= & -\int_{-\infty}^{\infty} e^{\xi}(\lambda-\beta) n d \xi+\int_{-\infty}^{\infty} e^{\xi} \frac{b}{k+1} v^{k+1} d \xi \\
& +\frac{3 a k-b}{2} \int_{-\infty}^{\infty} e^{\xi} v_{x}^{2} v^{k-1} d \xi+\frac{(k-1)(a k-b)}{2} \int_{-\infty}^{\infty} e^{\xi} v_{x}^{3} v^{k-2} d \xi
\end{aligned}
$$

If $b=a k$ and $k$ is a positive odd number, we obtain

$$
\frac{d}{d t} L_{+}(t)+(\lambda-\beta) L_{+}(t)>0,
$$

which is equivalent to the inequality

$$
\frac{d\left[L_{+}(t) e^{(\lambda-\beta) t}\right]}{d t}>0 .
$$

Hence $L_{+}(t)>0, t \in[0, T)$. 
Similarly, we have

$$
\frac{d\left[-L_{-}(t) e^{(\lambda+\beta) t}\right]}{d t}>0
$$

Thus, $L_{-}(t)<0, t \in[0, T)$.

If $k=1,0<b<3 a$, we derive that (4.8) and (4.9) still hold true.

We give the estimates for curve $p\left(t, b_{0}\right)$. Using the assumption $k=1, b=2 a$ and (3.4) yields

$$
\|v\|_{L^{\infty}} \leq\|v\|_{H^{1}} \leq e^{-\lambda t}\left\|v_{0}\right\|_{H^{1}} .
$$

Taking $x=b_{0}$ in (3.8) and integrating (3.8) on $[0, t]$, we come to the estimate

$$
\begin{aligned}
p\left(t, b_{0}\right) & =b_{0}+\int_{0}^{t} a v(\tau, p) d \tau+\beta t \\
& \leq \frac{1}{\lambda} C_{5}+b_{0}+\beta t
\end{aligned}
$$

We conclude from (4.2) that

$$
L_{+}(t)=2 e^{p\left(t, b_{0}\right)} v\left(t, p\left(t, b_{0}\right)\right) \leq C_{3} e^{(\beta-\lambda) t}
$$

Similar to the derivation in (4.11), we have

$$
\begin{aligned}
p\left(t, a_{0}\right) & =a_{0}+\int_{0}^{t} a v(\tau, p) d \tau+\beta t \\
& \geq-\frac{1}{\lambda} C_{5}+a_{0}+\beta t
\end{aligned}
$$

which, combining with (4.4), implies

$$
\left|L_{-}(t)\right| \leq C_{4} e^{-(\beta+\lambda) t}
$$

If $k=1, b=\frac{a}{2}$, it is deduced from (3.11) that $\|v\|_{L^{\infty}} \leq e^{-\lambda t}\left\|v_{0}\right\|_{H^{2}}$. Similarly, we establish (4.12) and (4.14).

Remark 4.1 If $\operatorname{supp} v_{0}(x) \subset\left[a_{0}, b_{0}\right]$ in (1.1), then $n=\left(1-\partial_{x}^{2}\right) v(t, x)$ satisfies supp $n \subset$ $\left[p\left(t, a_{0}\right), p\left(t, b_{0}\right)\right]$. Indeed, $v$ does not have compact support. Also $v(t, x)$ is positive if $x \rightarrow \infty$ and $v(t, x)$ is negative if $x \rightarrow-\infty$.

\section{Acknowledgements}

We are grateful to the anonymous referees for a number of valuable comments and suggestions.

\section{Funding}

The project is supported by Science Foundation of North University of China (No. 2017030, No. 13011920) and the National Natural Science Foundation of P.R. China (No. 11471263). 
Availability of data and materials

Not applicable.

\section{Competing interests}

The authors declare that they have no competing interests.

\section{Consent for publication}

All authors read and approved the final version of the manuscript.

Authors' contributions

All authors contributed equally in this paper. All authors read and approved the final manuscript.

\section{Author details}

'Department of Securities and Futures, Southwestern University of Finance and Economics, Chengdu, China. 2 Department of Mathematics, Southwestern University of Finance and Economics, Chengdu, China. ${ }^{3}$ Department of Mathematics, North University of China, Taiyuan, China.

\section{Publisher's Note}

Springer Nature remains neutral with regard to jurisdictional claims in published maps and institutional affiliations.

\section{Received: 3 July 2019 Accepted: 11 October 2019 Published online: 21 October 2019}

\section{References}

1. Constantin, A.: Global existence of solutions and breaking waves for a shallow water equation: a geometric approach. Ann. Inst. Fourier (Grenoble) 50, 321-362 (2000)

2. Fu, Y., Qu, C.Z.: Well-posedness and wave breaking of the degenerate Novikov equation. J. Differ. Equ. 263, 4634-4657 (2017)

3. Gao, Y., Li, L., Liu, J.G.: A dispersive regularization for the modified Camassa-Holm equation. SIAM J. Math. Anal. 50 2807-2838 (2018)

4. Guo, Z.G.: On an integrable Camassa-Holm type equation with cubic nonlinearity. Nonlinear Anal., Real World Appl. 34, 225-232 (2017)

5. Guo, Z.G., Li, X.G., Yu, C.: Some properties of solutions to the Camassa-Holm-type equation with higher order nonlinearities. J. Nonlinear Sci. 28, 1901-1914 (2018)

6. Himonas, A., Holliman, C., Kenig, C.: Construction of 2-peakon solutions and ill-posedness for the Novikov equation. SIAM J. Math. Anal. 50, 2968-3006 (2018)

7. Himonas, A., Mantzavinos, D.: An ab-family equations with peakon traveling waves. Proc. Am. Math. Soc. 144, 3797-3811 (2016)

8. Himonas, A., Thompson, R.: Persistence properties and unique continuation for a generalized Camassa-Holm equation. J. Math. Phys. 55, 091503 (2014)

9. Hu, Q.Y., Qiao, Z.J.: Global existence and propagation speed for a generalized Camassa-Holm model with both dissipation and dispersion (2015) arXiv:1511.03325v1

10. Lai, S.Y., Wu, Y.H.: A model containing both the Camassa-Holm and Degasperis-Procesi equations. J. Math. Anal. Appl. $374,458-469(2011)$

11. Li, M.G., Zhang, Q.T.: Generic regularity of conservative solutions to Camassa-Holm type equations. SIAM J. Math. Anal. 49, 2920-2949 (2017)

12. Mi, Y.S., Liu, Y., Guo, B.L., Luo, T.: The Cauchy problem for a generalized Camassa-Holm equation. J. Differ. Equ. 266, 6739-6770 (2019)

13. Ming, S., Lai, S.Y., Su, Y.Q.: The Cauchy problem of a weakly dissipative shallow water equation. Appl. Anal. 98, 1387-1402 (2019)

14. Molinet, L.: A Liouville property with application to asymptotic stability for the Camassa-Holm equation. Arch. Ration. Mech. Anal. 230, 185-230 (2018)

15. Novruzov, E., Hagverdiyev, A.: On the behavior of the solution of the dissipative Camassa-Holm equation with the arbitrary dispersion coefficient. J. Differ. Equ. 257, 4525-4541 (2014)

16. Tu, X.Y., Liu, Y., Mu, C.L.: Existence and uniqueness of the global conservative weak solutions to the rotation Camassa-Holm equation. J. Differ. Equ. 266, 4864-4900 (2019)

17. $\mathrm{Wu}, \mathrm{X} .:$ On the finite time singularities for a class of Degasperis-Procesi equations. Nonlinear Anal., Real World Appl. 44, 1-17 (2018)

18. Yan, W., Li, Y.S., Zhang, Y.M.: Global existence and blow-up phenomena for the weakly dissipative Novikov equation. Nonlinear Anal. 75, 2464-2473 (2012)

19. Zhang, L., Liu, B.: On the Cauchy problem for a class of shallow water wave equations with $(k+1)$-order nonlinearities J. Math. Anal. Appl. 445, 151-185 (2017)

20. Zhou, Y., Chen, H.P.: Wave breaking and propagation speed for the Camassa-Holm equation with $k \neq 0$. Nonlinear Anal., Real World Appl. 12, 1875-1882 (2011) 